\title{
Practice, systems and technology for seniors
}

\author{
David Prendergast $\cdot$ Simon Roberts
}

Published online: 15 July 2008

(C) Springer-Verlag 2008

\begin{abstract}
This paper explores how research teams in Intel's Digital Health Group are using ethnography to identify 'designable moments'-spaces, times, objects, issues and practices which suggest opportunities for appropriate interventions. It argues that technology innovation should aim to incorporate the views, experiences and practices of users from the start of the design process to support independent living and develop culturally sensitive enhancements that contribute towards wellbeing and a life of quality for local older populations.
\end{abstract}

\section{Keywords Ageing $\cdot$ Healthcare $\cdot$ Ethnography ·} Design

\author{
'When thinking about how practices evolve, it \\ becomes clear that relations between material objects \\ and associated images and forms of competence are \\ of defining importance'. \\ (Shove and Pantzer 4:43) \\ 'I don't want to use a walking stick, it will make me \\ look and feel old' \\ (Vera, 76, Germany)
}

The Product Research and Incubation (PRI) division of the Digital Health Group at Intel is chartered with understanding people's health needs and aspirations worldwide across the continuum of care. Understanding their daily practices, processes, values and systems helps us to invent

D. Prendergast $(\bowtie) \cdot S$. Roberts

Digital Health, Intel Corp, Collinstown Industrial Estate, Leixlip, Ireland

e-mail: David.K.Prendergast@Intel.Com and test tomorrow's health and wellness technologies and usage models. The PRI teams in Ireland and USA are currently involved in a number of local and global projects exploring how technology innovation can help to support independent living practice and develop culturally and contextually appropriate enhancements that contribute towards wellbeing and a life of quality for older people.

Intel's ongoing Global Ageing Experience (GAE) Project and its recent Material Culture and Ageing in Ireland (MCI) study, both utilise a practices-based approach to research. The GAE study of the everyday lives of older people in 12 countries around the world is already producing substantial insights into the interplay between activity, social networks, health and household routines and perceptions of ageing. To complement this cross national baseline research, several 'deeper dive' projects have also commenced building on the above initiatives. One of these, Community Supports for Ageing, is a long term engagement with an active retirement group and the wider community in a rural village in County Meath, Ireland to develop health and wellbeing technologies sensitive to the local cultural setting and individual practices. This project uses a variety of methods to explore different scales from interviews with major gatekeepers and system level service providers, to a community survey carried out by older volunteers, to participant observation in the active retirement group and wider community. A small internet cafe has recently been installed in the local third age centre which will provide us with the opportunity to observe patterns of internet usage and modes of learning amongst seniors in the area. At the core of the study is intensive household research with older people and an ongoing series of collaborative design exercises and workshops between members of the local community and the multidisciplinary Intel team. 
The research is attempting to incorporate the views and experiences of users from the start of the design process. The research teams are using ethnography to identify 'designable moments' - spaces, times, objects, issues and practices which suggest opportunities for appropriate interventions. Given that this research is being conducted by a high-tech company the temptation is, of course, to use the research to leap to a technology innovation or solution. However, we are attempting to resist the allure of the 'solution' as an automated response to the 'designable moments' we have identified. This is highly unlikely to be an indefinite delay, but one that is informed by another resistance-a resistance to the idea that ethnography can lead, in itself, to designs or products that are adopted unproblematically.

The view that technology for elders should be informed by a bottom-up, person-centred approach to research and innovation is scarcely groundbreaking. Equally, the idea of technology adoption as a process patterned by local sociocultural ideas, competencies and practices is not new either [5]. However, two streams of recent writing on design and practice provide us a new way to think about the passage from ethnography to design or technology innovation. These, in turn, might reshape the way a technology company such as Intel thinks about what it can offer to ageing populations worldwide.

The first stream is that of Paul Dourish and his paper 'Implications for Design' [2]. This is a rich commentary on ethnography and HCI design, which picks apart some of the tensions inherent in the activities we are concerned with. One such tension is the tendency of ethnography to place the user outside of the design process (the ethnographer becomes the gatekeeper of the research, the designer that of the 'design'). Another, alluded to above, is the desire, the need, to see research as valuable only in as far is supports design. However, in response to this, Dourish suggests:

'Sometimes, after all, the most effective outcome of a study might be to recommend what should not be built rather that to recommend what should. More to the point, an analysis of the cultural and social organization of some particular setting or occasion is often best articulated independently of specific systems, technologies, or design briefs.'

(Dourish 2006: 545)

This is a sentiment that we are trying to adopt as a practical maxim as our long-term research and innovation process develops. Elsewhere in the paper Dourish discusses the role of ethnography in terms of cultural practice. He draws on the work of Suchman [6] to suggest that we should be trying to 'respect and amplify local practices rather than to represent it for the purpose of design' (ibid). So, in summary, the work of Dourish comes with warnings about ethnography's tendency to exclude the 'user', despite its claims to the contrary. It also warns of the dangers of underplaying the importance of what we should not be doing or designing. It goes some way to putting the idea of practice at the centre of the ethnographic endeavour.

Our thinking about practice has been influenced by the recent work of Elizabeth Shove and Mika Pantzer [3, 4] and Alan Warde [7]. This encourages us to think of practice as being about routinised behaviour which consists of several elements interconnected to one another: forms of bodily activity, forms of mental activities, 'things' and their use and background knowledge in the form of understanding, know-how, emotions and motivational knowledge [1, 7]. For Shove and Pantzer practice represents the 'active integration of materials, meanings and forms of competence' and to attend to the relations between materials, meanings and competences [4]. Indeed using the example of the recent 'invention' of Nordic walking, they argue 'products alone have no value. They do so only when integrated into practice and...forms of competence and meaning' (ibid: 57).

Taken together, the perspective of Dourish and the above insights into practice are valuable for a number of reasons. They challenge the assumption that an 'object', thing or device is the natural outcome of our work. They force us to think about what we might need to do in addition to design technologies or objects to support lives of quality for older people, and therefore encourages us to locate our problem, and thus our research, beyond the question of design. It compels us to think about our task as one of 'system builders' where technology might be an enabler or lubricant but, in the final analysis, only ever a bit part player.

So in terms of defining research scopes and thinking about implementation of research projects, this perspective invites us to shift our attention to:

- How we bring seniors as close to the research and 'ideation' (the implications for design) as possible.

- What are people trying to achieve through their daily activities? For example, maintenance of mobility, public and personal identity work, cognitive acuity or demonstrations of competence.

- How are objects used to support practices and forms of competence and, in turn, how can we think less about designing objects or technologies but instead aid in the innovation of practices that technologies support?

- What constellations of meanings surround certain objects and how does these affect their incorporation, or otherwise, into daily practices?

- What we have to do in addition to research to give innovations and designs traction in the 'real world' of seniors. In particular what are the service and product ecosystems and networks with which we need to work? 


\section{Concluding remarks: implications for research with older people}

In terms of our current ageing related research agenda, and how this in turn informs design and system engineering, this approach suggests the following:

- Not thinking just in terms of new product innovations, but innovations in practice.

- Accepting that whilst producers may provide all the constituent elements that they can (for example, image and materials), it is how all the elements are bought together through practice that matters.

- Recognising that practices are dynamic in that they require continual reproduction. Whilst producers such as technology companies or device manufacturers may provide and promote a number of the elements, they can not make the practice happen. For example, we cannot ensure walking with a stick or wearing a home alarm pendant happens - that's up to the practitioners. What we can do is ensure that research highlights, and works with, existing meaningful social practice.
- Acknowledging the dangers surrounding technologies or services that deny agency or ignore, render obsolete, or deskill practices of importance to older people.

\section{References}

1. Blyth, S., Roberts, S.: Re-thinking market research. Paper presented at the MRS Conference 2005, London (2005)

2. Dourish, P.: Implications for design. In: Proceedings of CHI 2006, April 22-28, pp. 541-550 (2006)

3. Shove, E., Watson, M., Ingram, J.: Designing and consuming: objects, practices and processes. Cultures of consumption programme. ESRC \& AHRB (2005)

4. Shove, E., Pantzer, M.: Consumers, producers and practices: understanding the invention and reinvention of nordic walking. J. Consum. Cult. 5(1), 43-64 (2005)

5. Silverstone, R.: Consuming technologies: media and information in domestic spaces. Routledge and Kegan Paul, London (1992)

6. Suchman, L.: Anthropology as "brand": reflections on corporate anthropology. Department of Sociology, Lancaster University, UK. http://www.lancs.ac.uk/fass/sociology/papers/suchmananthropologyas-brand.pdf (2000)

7. Warde, A.: Consumption and theories of practice. J. Consum. Pract. 5(2), 131-153 (2005) 